\title{
On Exact Conservation for the Euler Equations with Complex Equations of State
}

\author{
J. W. Banks ${ }^{1, *}$ \\ 1 Center for Applied Scientific Computing, Lawrence Livermore National Lab- \\ oratory, Livermore, California, 94551
}

\begin{abstract}
Conservative numerical methods are often used for simulations of fluid flows involving shocks and other jumps with the understanding that conservation guarantees reasonable treatment near discontinuities. This is true in that convergent conservative approximations converge to weak solution and thus have the correct shock location. However, correct shock location results from any discretization whose violation of conservation approaches zero as the mesh is refined. Here we investigate the case of the Euler equations for a single gas using the Jones-Wilkins-Lee (JWL) equation of state. We show that a quasi-conservative method can lead to physically realistic solutions which are devoid of spurious pressure oscillations. Furthermore, we demonstrate that under certain conditions, a quasi-conservative method can exhibit higher rates of convergence near shocks than a strictly conservative counterpart of the same formal order.
\end{abstract}

AMS subject classifications: 65N08, 35L60, 35L65, 76N15

Key words: Euler equations, complex EOS, JWL EOS, Godunov methods

\section{Introduction}

The use of conservative schemes for simulations of solutions to hyperbolic conservation laws in the presence of discontinuities is ubiquitous. The reasons draw largely from the fact that convergent conservative schemes are known to converge to weak solutions in the presence of shocks by the Lax-Wendroff theorem [1]. On the other hand, non-conservative schemes can converge to non-weak solutions which violate the integral conservation equations [2]. However, weak solutions which violate physical properties, such as positivity of density or entropy satisfaction, are equally as troublesome as non-weak solutions and there is very little theory guaranteeing convergence for nonlinear systems to the appropriate "vanishing viscosity" solution (one notable exception is the random choice method of Glimm [3]). It is interesting then that so much emphasis has been placed on exact conservation rather than rapid convergence to the relevant vanishing viscosity solution.

${ }^{*}$ Corresponding author. Email addresses: banks20@llnl.gov (J.W. Banks) 
One prototypical physical system described by hyperbolic conservation laws is that of gas dynamics. Here the governing system is the Euler equations and for cases where the equation of state is sufficiently nonlinear, issues relating to exact conservation rise very much to the surface. The classical realization of these problems is unphysical oscillations, particularly in the pressure, arising near material interfaces in multi-material flows [4-10]. Somewhat less appreciated is the fact that the same type of behavior can be exhibited for single component flows with complicated equations of state [11].

The multi-component case has been studied extensively and many non-conservative or quasi-conservative schemes have been proposed and used to great effect in that context. Nonlinearities in the equation of state result when sharp material interfaces are smeared through the use of a capturing scheme. To remedy this, the paper by Quirk and Karni [5] for instance, relies on a non-conservative primitive formulation and adds source terms which restore conservation where possible. Other choices that have been made include the use of a hybrid approach that relies on the conservative update whenever possible but uses the primitive update near interfaces [4], conservative approximations which add source terms to break conservation as required near material interfaces $[9,10]$, and special advection rules for particular components of the equation of state $[6,7]$. All of these approaches seek to realize physically realistic treatment of the material interface through the use of non-conservative schemes and rely on the fact that the poor behavior exhibited by capturing schemes is limited to a small region about the material interface.

In [11] the authors show that poor behavior is not limited to material interfaces and problems can occur for single component flows with sufficiently nonlinear equations of state. In that paper, the authors present a new numerical method to treat such flows. Their scheme relies on a specific class of Riemann solver, a specific equation of state, and in the end introduces special advection rules for the adiabatic exponent which amounts to a modification of the equation of state. Their approach does seems to have some efficacy when it is applicable, but alterations of the equation of state can lead to inconsistent numerical schemes and great care must be used. Without assurances that the numerical system is consistent with the original PDE as well as convergent, this scheme also faces obstacles in terms of application of the Lax-Wendroff theorem.

The state of matters then seems to be that the classical, that is to say consistent and conservative, schemes may produce physically unrealistic results; especially near contacts. As a result, even though some theoretical benefit is achieved through the use of fully conservative schemes, they can produce approximations which are not useful and so modifications to these schemes are developed. On one hand there is the class of schemes which modify the governing equations and thus risk inconsistency [11]. On the other hand there is the class of schemes which modify conservation with the obvious associated risks [4-10]. It is not at all clear that these approaches are entirely different and it may be the case that the actual numerical approximations are quite similar.

In the current paper we will demonstrate the poor behavior of one particular classical conservative scheme when applied to a simple Riemann problem with a single JonesWilkins-Lee (JWL) gas. The choice of the JWL equation of state is motivated by high 
explosives applications, but many other real gas equations of state would exhibit similar behavior. For this problem, the global convergence character of the solution is demonstrated to be dominated by the convergence character near the contact and somewhat surprisingly the shock is seen to be incorrectly located by an increasing number of grid cells as the grid spacing is reduced. To contrast this, the quasi-conservative scheme of [9] is modified so that the non-conservative source term is active whenever the acoustic characteristic field is not dominant. This type of switching mechanism yields the property that as the grid is refined, the error in energy conservation approaches zero. As a result, the approximations approach a conservative solution and, because the method is consistent, the Lax-Wendroff theorem is applicable. We call such schemes quasi-conservative schemes. The primary benefits of this new scheme are its applicability to any equation of state, any Riemann solver, and higher dimensions without the use of dimensional splitting. Interestingly, the new scheme is shown to produce more rapid convergence near the shock of the example Riemann problem than a fully conservative counterpart. High convergence rates may be important in many applications. For instance in a reacting flow, stiff source terms can require highly accurate flow fields in order to faithfully reproduce the dynamics of the physical system.

The remainder of this paper is organized as follows. Section 2 presents the governing equations including the JWL equation of state which we use. A solution strategy for the Riemann problem with general convex equations of state is presented in section 3 . The numerical methods used in the paper are developed in section 4 . Numerical results for the application of these schemes to a Riemann problem are presented in section 5. A more practical example of the associated issues is presented in section 6 and concluding remarks are made in section 7 .

\section{Governing equations}

The governing equations under consideration in this paper are the one-dimensional Euler equations for a single component. These equations can be viewed as the system of conservation laws

where

$$
\frac{\partial}{\partial t} \mathbf{u}+\frac{\partial}{\partial x} \mathbf{f}(\mathbf{u})=0,
$$

$$
\mathbf{u}=\left[\begin{array}{c}
\rho \\
\rho u \\
\rho E
\end{array}\right], \quad \mathbf{f}(\mathbf{u})=\left[\begin{array}{c}
\rho u \\
\rho u^{2}+p \\
u(\rho E+p)
\end{array}\right]
$$

The variables have the usual meanings with $\rho$ being the density, $u$ the velocity, $E$ the total energy per unit mass, and $p$ the pressure. Here the total energy for the fluid is given by

$$
E=e+\frac{1}{2} u^{2},
$$

where $e=e(\rho, p)$ is the specific internal energy which is specified through an equation of state (EOS). The equations are assumed to have been rendered dimensionless using 
suitable scalings of the variables. For this work, emphasis is place on non-ideal equations of state and a Jones-Wilkins-Lee (JWL) EOS is chosen. This EOS is given by

$$
e(\rho, p)=\frac{p v}{\omega}-\mathcal{F}(v)+\mathcal{F}\left(v_{0}\right)
$$

where $v=\frac{1}{\rho}$ and $p$ are the specific volume and pressure respectively, and $v_{0}$ is a reference specific volume. Such an EOS is often encountered when modeling high explosives. The use of this specific EOS is not critical and is just one example where the associated problems might arise. This choice was made to make the discussion concrete, but other forms such as Mie-Grüneisen, Van der Waals, or others could have been considered. In the JWL forms, $\omega$ is the Grüneisen coefficient, and $\mathcal{F}(v)$, the stiffening function, is given by

$$
\mathcal{F}(v)=A\left(\frac{v}{\omega}-\frac{1}{R_{1}}\right) \exp \left(-R_{1} v\right)+B\left(\frac{v}{\omega}-\frac{1}{R_{2}}\right) \exp \left(-R_{2} v\right),
$$

where $A, B, R_{1}$ and $R_{2}$ are constants for a given material [12]. Equation (2.2) represents the mechanical EOS but the full JWL model involves a thermal equation of state as well. Both the mechanical and thermal equations might be considered when modeling mixtures of multiple materials for example, but for this single gas case the mechanical EOS is sufficient. Notice that the JWL form in (2.2) and (2.3) includes as a special case the ideal gas EOS when $\mathcal{F}(v)=0$. For the simulations presented in this paper, the particular choice for the EOS parameters is shown in table 1 which corresponds roughly to the solid used for some condensed-phase explosive models [12].

\begin{tabular}{|c||c|c|c|c|c|c|}
\hline \hline parameter & $A$ & $B$ & $R_{1}$ & $R_{2}$ & $\omega$ & $v_{0}$ \\
\hline value & 692.5067 & -0.044776 & 11.3 & 1.13 & 0.8938 & 0.5 \\
\hline \hline
\end{tabular}

Table 1: Values for the parameter associated with the EOS (2.2).

\section{Exact solution of Riemann problem with convex EOS}

One particularly important solution to (2.1) is the solution of the Riemann problem. The exact solution to the Riemann problem for convex EOS has been investigated for example in [13-15] but it is useful to give a brief outline of a complete numerical strategy. Given left and right primitive states $\mathbf{w}_{L}=\left[\rho_{L}, u_{L}, p_{L}\right]^{T}$ and $\mathbf{w}_{R}=\left[\rho_{R}, u_{R}, p_{R}\right]^{T}$ respectively, the solution consists of left and right acoustic waves and a center contact. Each acoustic wave can be either a shock or rarefaction and thus there are four possible configurations. One such configuration is shown schematically in figure 1. As in many previous works, the "star" superscript is used to denote the region between the two acoustic waves.

The solution strategy used here, which is similar to that discussed in [14] and [15], involves the solution of a nonlinear equation for the center pressure $p^{*}$. To ensure robustness of the solver, this is realized numerically as a bisection iteration. At each iteration, 


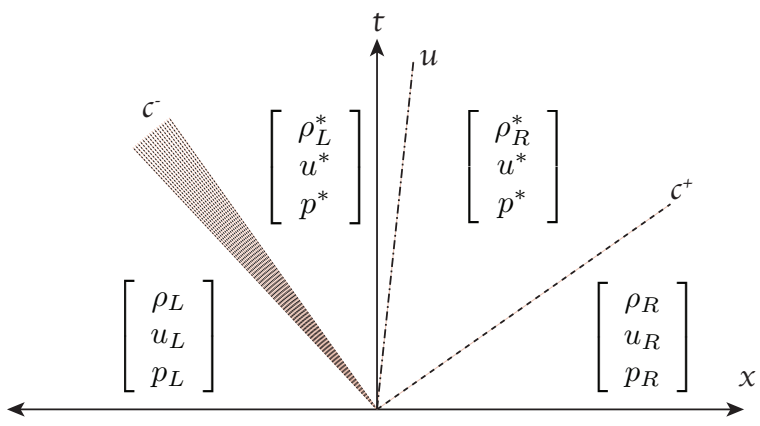

Figure 1: Typical structure of the Riemann problem in $x-t$ space. Here the left and right acoustic waves are labeled with $c^{-}$and $c^{+}$respectively and the contact path with $u$. In this configuration, the solution consists of a left rarefaction and a right shock. The $*$-state lies between the acoustic waves with the pressure and velocity remaining constant across the contact.

the approximation of $p^{*}$ determines the wave structure from the four possibilities. For instance, if $p^{*}>p_{L}$ then the $c^{-}$wave is a shock. If $p^{*} \leq p_{L}$ then the $c^{-}$wave is a rarefaction. Similar reasoning determines the character of the $c^{+}$wave. Once the solution structure is determined, a provisional solution is computed through each acoustic wave. This provisional solution satisfies the appropriate Riemann invariants through the left and right acoustic waves and has constant pressure $p^{*}$ through the contact. However, the velocities on either side of the contact may not agree as they must in the final solution. If the calculated values of $u^{*}$ on the left and right of the contact match (perhaps to a tolerance), then the iteration is halted. Otherwise a new approximation for $p^{*}$ is chosen and the iteration continues.

\subsection{Solution through a shock}

Determination of the solution across a shock is straight forward. The Rankine-Hugoniot jump conditions are given for example in [16] as

$$
\begin{aligned}
\rho_{1}\left(D-u_{1}\right) & =\rho_{0}\left(D-u_{0}\right) \\
\rho_{1}\left(D-u_{1}\right)^{2}+p_{1} & =\rho_{0}\left(D-u_{0}\right)^{2}+p_{0} \\
h_{1}+\frac{1}{2}\left(D-u_{1}\right)^{2} & =h_{0}+\frac{1}{2}\left(D-u_{0}\right)^{2}
\end{aligned}
$$

where $\rho_{0}, u_{0}, h_{0}$ is the state ahead of the shock, $\rho_{1}, u_{1}, h_{1}$ is the state behind the shock, $h=e(\rho, p)+\frac{p}{\rho}$ is the enthalpy, and $D$ is the shock speed. Enforcing that $p_{1}=p^{*}$ gives

$$
e\left(\rho_{1}, p^{*}\right)+\frac{p^{*}}{\rho_{1}}+\frac{\rho_{0}\left(p^{*}-p_{0}\right)}{2 \rho_{1}\left(\rho_{1}-\rho_{0}\right)}=e\left(\rho_{0}, p_{0}\right)+\frac{p_{0}}{\rho_{0}}+\frac{\rho_{1}\left(p^{*}-p_{0}\right)}{2 \rho_{0}\left(\rho_{1}-\rho_{0}\right)} .
$$

Equation (3.2) is solved for $\rho_{1}$ using a Newton iteration and the remainder of the solution follows trivially. Included in this solution is the shock speed $D$. 


\subsection{Solution through a rarefaction}

Numerical approximation through a rarefaction wave is somewhat more involved but conceptually straightforward. Rarefactions are isentropic implying that the sound speed $a$ depends only on the density. Determination of the sound speed $a$ will be discussed in section 3.3. This implies $a^{2}=\frac{\partial p}{\partial \rho}$ and so

$$
\int[a(\rho, p(\rho))]^{2} d \rho=\int d p
$$

Starting from the state $\left[\rho_{0}, u_{0}, p_{0}\right]^{T}$, the solution is determined in a Runge-Kutta like strategy $[17]$

$$
p_{k+1} \approx p_{k}-\frac{\Delta \rho}{6}\left(\left[a\left(\rho_{k}, \hat{p}_{1}\right)\right]^{2}+2\left[a\left(\rho_{k+1 / 2}, \hat{p}_{2}\right)\right]^{2}+2\left[a\left(\rho_{k+1 / 2}, \hat{p}_{3}\right)\right]^{2}+\left[a\left(\rho_{k+1}, \hat{p}_{4}\right)\right]^{2}\right)
$$

where

$$
\begin{array}{ll}
\hat{p}_{1}=p_{k}, & \hat{p}_{2}=p_{k}-\frac{\Delta \rho}{2}\left[a\left(\rho_{k+1 / 2}, \hat{p}_{1}\right)\right]^{2}, \\
\hat{p}_{3}=p_{k}-\frac{\Delta \rho}{2}\left[a\left(\rho_{k+1 / 2}, \hat{p}_{2}\right)\right]^{2}, & \hat{p}_{4}=p_{k}-\frac{\Delta \rho}{2}\left[a\left(\rho_{k+1}, \hat{p}_{3}\right)\right]^{2},
\end{array}
$$

and $\rho_{k+1 / 2}=\left(\rho_{k}+\rho_{k+1}\right) / 2$ for values of $k=0,1, \ldots, N$. A secant method is used to determines the value of $\Delta \rho$ so that $\left|p_{N}-p^{*}\right|<1 \times 10^{-12}$ using a fixed $N$ taken to be $N=10000$.

The velocity at $\rho_{k+1}$ is found using Riemann invariants (see [16]) which determine $u_{k+1}=u_{0}+\int_{\rho_{0}}^{\rho_{k+1}} \frac{a(\rho, p(\rho))}{\rho} d \rho$. Approximating this integral as before gives

$$
u_{k+1} \approx u_{k}-\frac{\Delta \rho}{6}\left(\frac{a\left(\rho_{k}, \hat{p}_{1}\right)}{\rho_{k}}+2 \frac{a\left(\rho_{k+1 / 2}, \hat{p}_{2}\right)}{\rho_{k+1 / 2}}+2 \frac{a\left(\rho_{k+1 / 2}, \hat{p}_{3}\right)}{\rho_{k+1 / 2}}+\frac{a\left(\rho_{k+1}, \hat{p}_{4}\right)}{\rho_{k+1}}\right) .
$$

To complete the rarefaction solution, the value for the similarity variable $\xi_{k}=\frac{x_{k}}{t}, t>0$ is determined by appealing to the second Riemann invariant and thus $\xi_{k} \approx u_{k}+a\left(\rho_{k}, p_{k}\right)$ for all $k$.

\subsection{Determination of the sound speed}

Sections 3.1 and 3.2 give methods to determine the solution through the two acoustic waves. The full solution for any convex EOS can then be determined through iteration on $p^{*}$ until the velocities on either side of the contact are in agreement. The sound speed $a=a(\rho, p)$ can be found by the basic methodology presented in $[9,18]$. For instance, given the $\operatorname{EOS} e=e(\rho, p)$, the square of the sound speed is

$$
a^{2}=\frac{\frac{p}{\rho^{2}}-\frac{\partial}{\partial \rho} e(\rho, p)}{\frac{\partial}{\partial p} e(\rho, p)} .
$$

Equation (3.4) can be found through an analysis of the eigen-structure of the flux Jacobian matrix from (2.1). 


\section{Numerical methods}

Although the exact solution to the Riemann problem can be found as in section 3 , it is of little practical use for more complex flows. Such a solution may be used as part of a numerical scheme which treats such flows, but more likely an approximate Riemann solver will be employed because the exact solver is computationally expensive. For this paper, the exact solver is used to obtain the exact solution of Riemann problems which are then used in the comparison of solution approximations obtained through other techniques. Detailed formulation of the numerical methods used here is the subject of other work $[9,10,18-20]$. However, we now provide a brief overview so that the basic approach is clear.

\subsection{A high-resolution Godunov method}

The high-resolution Godunov technique [21,22] has proven successful in approximating solutions of conservation systems such as (2.1). Schemes in this class evolve cell averages based on an integral formulation of (2.1) and use solution dependent switches to choose between high-order and low-order approximations. The schemes considered here achieve second order accuracy for sufficiently smooth flows and reduce to first order near solution extrema.

Discrete solutions will be found on the mesh defined by

$$
\mathcal{L}\left(\left[x_{a}, x_{b}\right], N\right)=\left\{x_{i} \mid x_{i}=x_{a}+i \Delta x, \Delta x=\left(x_{b}-x_{a}\right) / N, i=0,1, \ldots, N\right\},
$$

Also introduce the temporal discretization $t^{n}=n \Delta t, n=1, \ldots$. A second order approximation to $(2.1)$ is given by

$$
\mathbf{u}_{i}^{n+1}=\mathbf{u}_{i}^{n}-\frac{\Delta t}{\Delta x}\left(\mathbf{f}_{i+1 / 2}^{n+1 / 2}-\mathbf{f}_{i-1 / 2}^{n+1 / 2}\right)
$$

where $\mathbf{u}_{i}^{n} \approx \mathbf{u}\left(x_{i}, t^{n}\right)$ and $\mathbf{f}_{i+1 / 2}^{n+1 / 2} \approx \mathbf{f}\left(\mathbf{u}\left(x_{i+1 / 2}, t^{n+1 / 2}\right)\right)$. Notice that the point value at the cell center is a second order approximation to the cell average. The value $\mathbf{u}_{i+1 / 2}^{n+1 / 2}$ is determined as the solution to a Riemann problem, approximate or exact, at the cell face $x_{i+1 / 2}$. The left and right states to this Riemann problem are denoted $\mathbf{u}_{L, i+1 / 2}^{n+1 / 2}$ and $\mathbf{u}_{R, i+1 / 2}^{n+1 / 2}$ respectively.

In order to achieve high-order accuracy for smooth flows, a MUSCL type slope correction is employed [22,23]. As discussed in [9] and [10], it is important that this slope correction be performed in primitive quantities in order to avoid spurious oscillations. Let $\mathbf{w}=[\rho, u, p]^{T}$ be the vector of primitive quantities. In primitive form, equation (2.1) becomes

where

$$
\frac{\partial}{\partial t} \mathbf{w}+\mathbf{A} \frac{\partial}{\partial x} \mathbf{w}=0
$$

$$
\mathbf{A}=\left[\begin{array}{ccc}
u & \rho & 0 \\
0 & u & \frac{1}{\rho} \\
0 & a^{2} \rho & u
\end{array}\right]
$$


Taylor series expansions are performed in both space and time and the resulting derivatives are replaced with finite differences. As in [9] this gives

$$
\begin{aligned}
\mathbf{w}_{L, i+1 / 2}^{n+1 / 2} & =\mathbf{w}_{i}^{n}+\frac{1}{2} \mathbf{R}_{i}^{n}\left(\mathbf{I}-\frac{\Delta t}{\Delta x} \max \left\{0, \Lambda_{i}^{n}\right\}\right) \alpha_{i}^{n} \\
\mathbf{w}_{R, i+1 / 2}^{n+1 / 2} & =\mathbf{w}_{i+1}^{n}-\frac{1}{2} \mathbf{R}_{i+1}^{n}\left(\mathbf{I}+\frac{\Delta t}{\Delta x} \min \left\{0, \Lambda_{i+1}^{n}\right\}\right) \alpha_{i+1}^{n}
\end{aligned}
$$

where $\mathbf{A}=\mathbf{R} \Lambda \mathbf{R}^{-1}$ is the eigen-decomposition of the flux Jacobian $\mathbf{A}$. The max and min functions are applied to each eigenvalue in $\Lambda$. The quantity $\alpha_{i}^{n}$ is defined by $\alpha_{i}^{n}=$ $\operatorname{MinMod}\left(\alpha_{i,-}^{n}, \alpha_{i,+}^{n}\right)$,

$$
\begin{aligned}
& \alpha_{i,-}^{n}=\left(\mathbf{R}^{-1}\right)_{i}^{n}\left(\mathbf{w}_{i}^{n}-\mathbf{w}_{i-1}^{n}\right) \\
& \alpha_{i,+}^{n}=\left(\mathbf{R}^{-1}\right)_{i}^{n}\left(\mathbf{w}_{i+1}^{n}-\mathbf{w}_{i}^{n}\right),
\end{aligned}
$$

and MinMod is the minimum modulus function applied component-wise. Once the states $\mathbf{w}_{L, i+1 / 2}^{n+1 / 2}$ and $\mathbf{w}_{R, i+1 / 2}^{n+1 / 2}$ are obtained, a simple conversion from primitive to conservative variables is performed to give $\mathbf{u}_{L, i+1 / 2}^{n+1 / 2}$ and $\mathbf{u}_{R, i+1 / 2}^{n+1 / 2}$. To complete the description, we note that a Roe approximate Riemann solver for non-ideal EOS is used to solve inter-cell Riemann problems (see $[19,24]$ for details). Finally note that for all computations in this paper the time step is chosen using a CFL number 0.8 .

\subsection{Energy correction method}

As discussed in [11], conservative schemes such as equation (4.2) can produce unphysical behavior near contacts. For example, figure 2 shows numerical results for a contact only Riemann problem with $[\rho, u, p]^{T}=[1.5,1,2]$ for $x<1 / 2$ and $[\rho, u, p]^{T}=[1,1,2]$ for $x \geq 1 / 2$. Here we use the grid $\mathcal{L}([0,1], 100)$ (as defined in equation (4.1)), and show results at $t=0.1$. The exact solution for this problem is a contact with uniform pressure and velocity (UPV) for all time. The figure shows results for both the Godnov scheme of (4.2) as well as energy corrected scheme (4.6) with $s_{i}^{n}=1$ (note that the results with $s_{i}^{n}$ defined using (4.8) are indistinguishable from those with $s_{i}^{n}=1$ for this problem and are not presented). The fundamental problem, that the pressure and velocity oscillate un-physically, is similar to the problems motivating the work in [9] and [10] and so we introduce a similar energy correction source term.

The basic energy corrected scheme takes the form

$$
\mathbf{u}_{i}^{n+1}=\hat{\mathbf{u}}_{i}^{n+1}+s_{i}^{n} \Delta G_{i}^{n} .
$$

Here $\hat{\mathbf{u}}_{i}^{n+1}$ is a provisional solution determined by the usual Godunov update

$$
\hat{\mathbf{u}}_{i}^{n+1}=\mathbf{u}_{i}^{n}-\frac{\Delta t}{\Delta x}\left(\mathbf{f}_{i+1 / 2}^{n+1 / 2}-\mathbf{f}_{i-1 / 2}^{n+1 / 2}\right)
$$



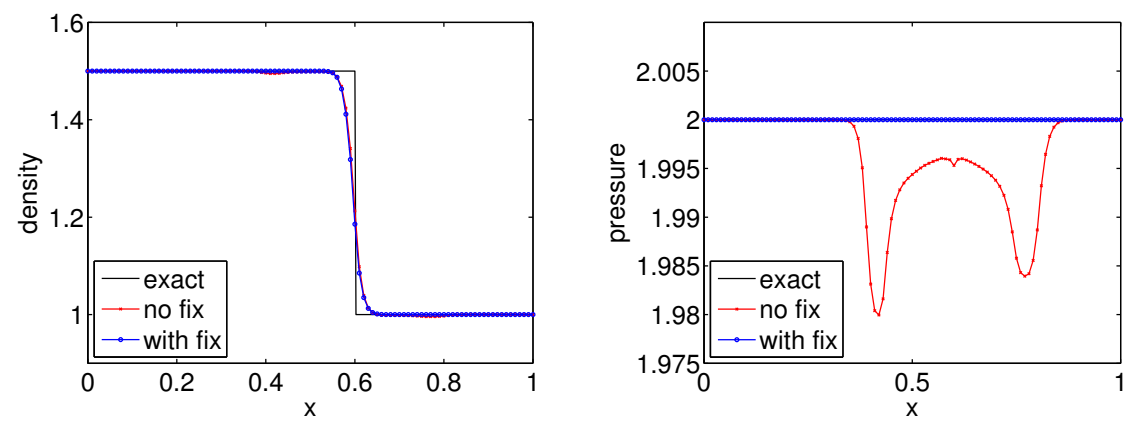

Figure 2: Numerical results for a contact only problem with the traditional Godunov scheme (4.2) as well as the scheme with energy corrected source term. Notice the deviation in pressure from UPV flow for the standard conservative scheme.

the source term $\Delta G_{i}^{n}$ is constructed to maintain UPV flow, and $s_{i}^{n}$ is a switch to be defined in section 4.3. In order to describe $\Delta G_{i}^{n}$ we perform the auxiliary update

$$
\tilde{\mathbf{u}}_{i}^{n+1}=\mathbf{u}_{i}^{n}-\frac{\Delta t}{\Delta x}\left(\tilde{\mathbf{f}}_{i+1 / 2}^{n+1 / 2}-\tilde{\mathbf{f}}_{i-1 / 2}^{n+1 / 2}\right)
$$

where $\tilde{\mathbf{f}}_{i \pm 1 / 2}^{n+1 / 2}$ are flux calculations using an artificially imposed UPV flow. For instance, the auxiliary flux $\tilde{\mathbf{f}}_{i+1 / 2}^{n+1 / 2}$ is determined from the Riemann problem with left and right states $\tilde{\mathbf{u}}_{L, i+1 / 2}^{n+1 / 2}$ and $\tilde{\mathbf{u}}_{R, i+1 / 2}^{n+1 / 2}$ which are the conservative states corresponding to the primitive states

$$
\tilde{\mathbf{w}}_{L, i+1 / 2}=\left[\begin{array}{c}
\rho_{L, i+1 / 2}^{n+1 / 2} \\
u_{i}^{n} \\
p_{i}^{n}
\end{array}\right], \quad \tilde{\mathbf{w}}_{R, i+1 / 2}=\left[\begin{array}{c}
\rho_{R, i+1 / 2}^{n+1 / 2} \\
u_{i}^{n} \\
p_{i}^{n}
\end{array}\right] .
$$

The source term in equation (4.6) is now defined by $\Delta G_{i}^{n}=\left[0,0, \Delta E_{i}^{n}\right]^{T}$ where

$$
\Delta E_{i}^{n}=\hat{\rho}_{i}^{n+1} e\left(\hat{\rho}_{i}^{n+1}, \hat{p}_{i}^{n+1}+\Delta p_{i}^{n}\right)-\hat{\rho}_{i}^{n+1} e\left(\hat{\rho}_{i}^{n+1}, \hat{p}_{i}^{n+1}\right)
$$

and

$$
\Delta p_{i}^{n}=p_{i}^{n}-\tilde{p}_{i}^{n+1}
$$

Notice that the Riemann problems in equation (4.7) must be solved using the same Riemann solver as was used in equation (4.6). Refer to figure 2 which shows the results for the contact only problem using the energy correction with $s_{i}^{n}=1$.

\subsection{Quasi-conservative acoustic switch}

In $[9,10]$ the non-conservative source term $\Delta G$ was activated only near material interfaces where two separate gasses contact one another. In the current context that switch is 

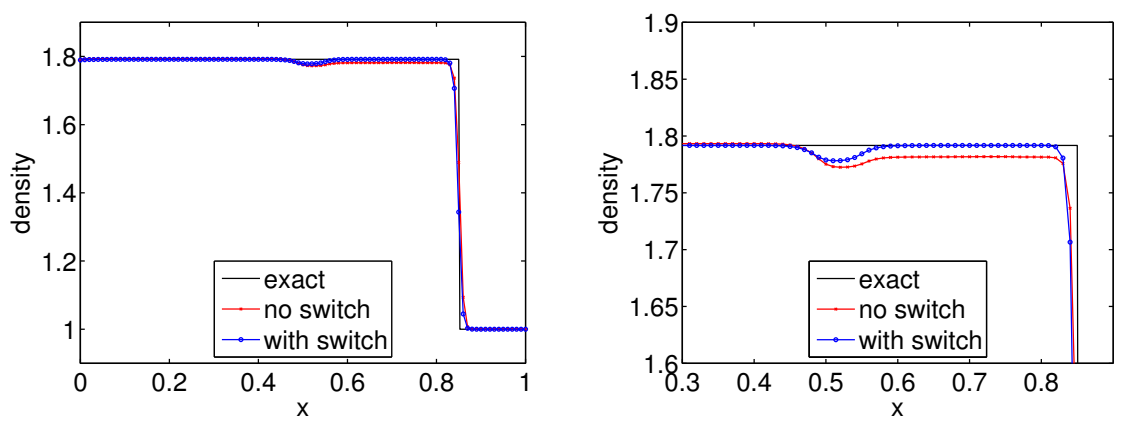

Figure 3: Approximations with and without the switch of equation (4.8). Both the full domain on the left and more detailed zoom on the right are show. Failing to turn off the source term near the shock results in a genuinely non-conservative approximation and convergence to a non-weak solution as shown in the figure.

changed so that the source term is active whenever the acoustic waves are not dominate. The term $s_{i}^{n}$ in equation(4.6) is used to implement this switch and is defined by

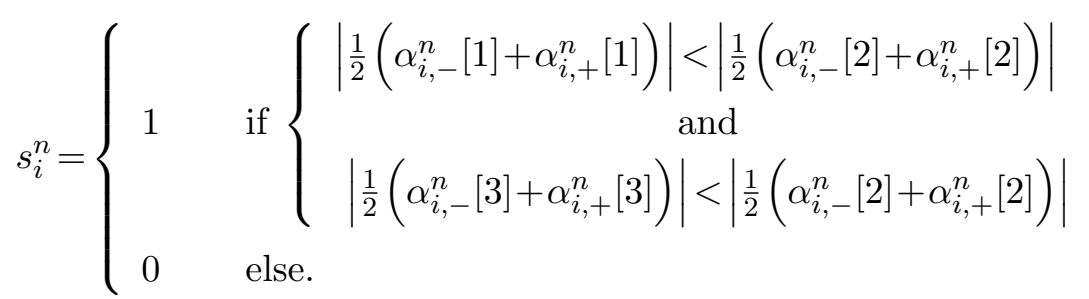

In equation (4.8), the notation $\alpha_{i,+}^{n}[\kappa]$ indicates the $\kappa$-component of the vector, and the eigen-decomposition has been performed in such as way that the first and last components of $\alpha$ correspond to the acoustic fields.

The reasoning to include this type of switch is to ensure that the non-conservative source term is turned off near shock waves. If the source term is active near shocks, a truly non-conservative scheme can result and convergence to non-weak solutions can be exhibited. To illustrate this type of error, figure 3 shows numerical results for the scheme with the acoustic switch $\left(s_{i}^{n}\right.$ as in (4.8)) as compared to results from the scheme with a globally active source term $\left(s_{i}^{n}=1\right)$ using $\mathcal{L}([0,1], 100)$. The initial conditions represent an exact shock jump in a JWL gas and are found as outlined in section 3.1 and set $[\rho, u, p]^{T}=[1.7917,1.3295,5]^{T}$ for $x<0.25$ and $[\rho, u, p]^{T}=[1,0,1]^{T}$ for $x \geq 0.25$. The resulting shock travels to the right with speed 3.009 and results are presented at $t=0.2$. The figure illustrates that failing to turn off the non-conservative source term near shocks will produce, for this case, a genuinely non-conservative result. Further refinement reaffirms that the approximation is converging to a non-weak solution. On the other hand, when the switch of equation (4.8) is used, the evidence indicates that in the limit of vanishing grid spacing the integrated contribution of the source term vanishes. Table 2, for example, shows the size of the violation of conservation of total energy for both schemes as the computational mesh is refined. By using the acoustic switch mechanism of equation (4.8), 


\begin{tabular}{||c||c|c||}
\hline \hline$m$ & energy gain without switch & energy gain with switch \\
\hline \hline 101 & $3.1383 \mathrm{e}-2$ & $8.0240 \mathrm{e}-5$ \\
\hline 201 & $3.1399 \mathrm{e}-2$ & $4.4719 \mathrm{e}-5$ \\
\hline 401 & $3.1384 \mathrm{e}-2$ & $2.3977 \mathrm{e}-5$ \\
\hline 801 & $3.1389 \mathrm{e}-2$ & $1.2725 \mathrm{e}-5$ \\
\hline 1601 & $3.1384 \mathrm{e}-2$ & $6.5999 \mathrm{e}-6$ \\
\hline 3201 & $3.1382 \mathrm{e}-2$ & $3.4031 \mathrm{e}-6$ \\
\hline 6401 & $3.1381 \mathrm{e}-2$ & $1.8038 \mathrm{e}-6$ \\
\hline 12801 & $3.1381 \mathrm{e}-2$ & $9.6341 \mathrm{e}-7$ \\
\hline \hline
\end{tabular}

Table 2: Total energy gain by the scheme with and without the acoustic switch for a single shock Riemann problem where $m$ is the number of grid cells.

the violation of energy conservation approaches zero as the mesh is refined and so in the limit, a conservative result is obtained. We call such schemes quasi-conservative. For all subsequent results the acoustic switch in (4.8) is used to define $s_{i}^{n}$.

\section{$5 \quad$ Numerical results}

We now provide a simple numerical example to illustrate that the quasi-conservative scheme of (4.6) behaves favorably in relation to the conservative scheme of (4.2) in a number of important ways. First, (4.6) produces results which are devoid of oscillations in the pressure and velocity even near contacts while (4.2) does not. Secondly the convergence rates for the two methods is investigated near the shock of a Riemann problem. The quasi-conservative scheme (4.6) is found to converge as $O(\Delta x)$ while the conservative scheme (4.2) is found to converge at $O\left(\Delta x^{2 / 3}\right)$. Consider the Riemann problem $[\rho, u, p]^{T}=[0.5,0,1]^{T}$ for $x<0$ and $[\rho, u, p]^{T}=[1,0,2]^{T}$ for $x \geq 0$. Recall that the full model, including EOS parameters, is given in section 2. The computational domain for this investigation is given as $\mathcal{L}([-5,5], m)$, and numerical integration is carried out to a final time $t_{f}=1.5$. The exact solution is approximated to a small tolerance through the methods described in section 3 and is used as a basis for comparison as needed. For this case, the exact solution consists of a left moving shock, a left moving contact, and a right moving rarefaction wave.

The numerical approximation found by the conservative Godunov method (4.2) with $m=200$, is shown in figure 4. For this approximation, the pressure is not uniform in the $*$-region and the velocity seems to jump across the material contact. Figure 5 shows the solution to the same problem using the quasi-conservative scheme (4.6). Clearly the solution behavior across the contact in the $*$-regions is substantially better than the corresponding fully conservative results in figure 4. Also note that the shock lags behind the exact location for the fully conservative result, while the quasi-conservative result shows 

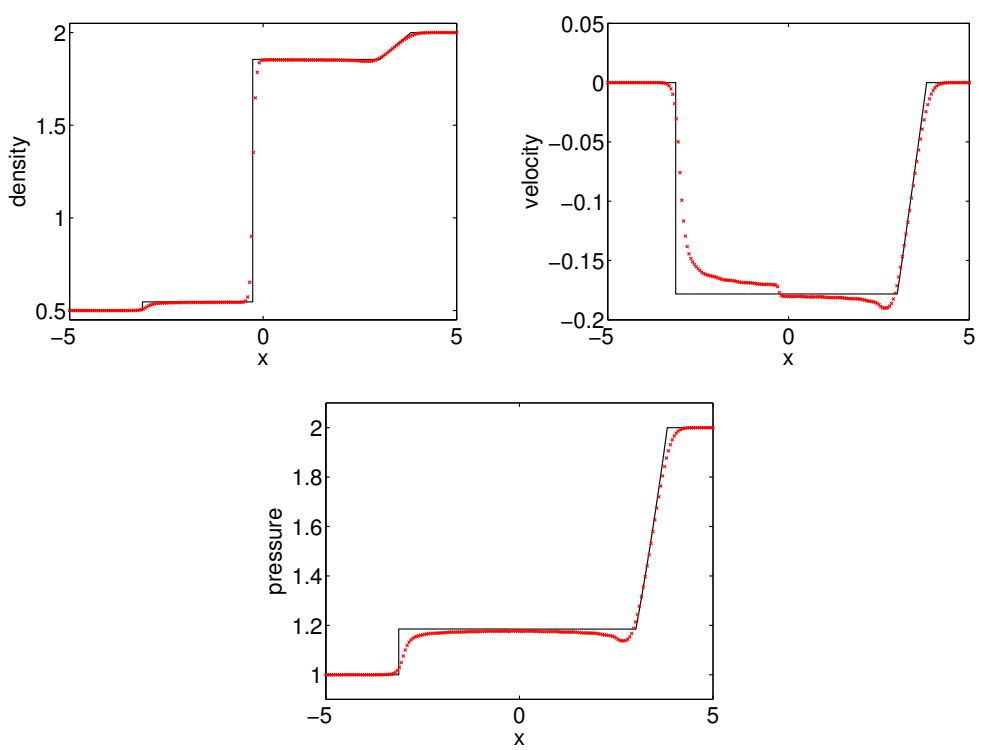

Figure 4: Exact solution for the Riemann (black line) and high-resolution Godunov approximation (red marks) at $t_{f}=1.5$. The computation is performed using $m=200$.
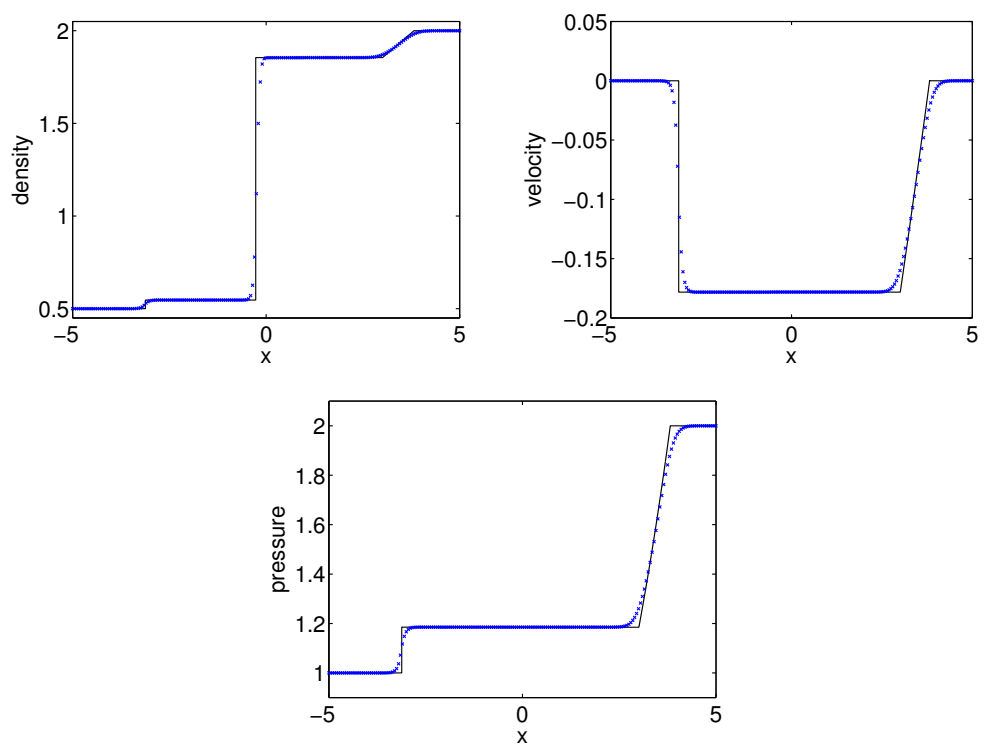

Figure 5: Exact solution for the Riemann problem (black line) and high-resolution quasi-conservative Godunov approximation (blue marks) $t_{f}=1.5$. The computation is performed using $m=201$ where $m$ indicates the number of grid cells for $x \in[-5,5]$. 
the approximate shock directly atop the exact location.

We now perform a convergence study and measure the $L_{1}$ convergence of the solution in the vicinity of the shock. The exact shock propagation velocity is found to be $D \approx-2.08$ and so at $t=1.5$ the shock location is $x \approx-3.11$. We calculate the $L_{1}$ error in the solution for $x \in[-3.2,-3]$ so that we measure convergence near the shock only. Note that the exact solution is found to much higher tolerances than the three decimals indicated above.

\begin{tabular}{||c||c|c||c|c||c|c||}
\hline \hline$m$ & $e_{\rho}(m)$ & rate & $e_{u}(m)$ & rate & $e_{p}(m)$ & rate \\
\hline \hline 201 & $3.88 \mathrm{e}-3$ & - & $1.47 \mathrm{e}-2$ & - & $1.54 \mathrm{e}-2$ & - \\
\hline 401 & $3.41 \mathrm{e}-3$ & 0.19 & $1.29 \mathrm{e}-2$ & 0.19 & $1.36 \mathrm{e}-2$ & 0.18 \\
\hline 801 & $2.84 \mathrm{e}-3$ & 0.26 & $1.07 \mathrm{e}-2$ & 0.27 & $1.13 \mathrm{e}-2$ & 0.27 \\
\hline 1601 & $1.78 \mathrm{e}-3$ & 0.67 & $6.70 \mathrm{e}-3$ & 0.68 & $7.10 \mathrm{e}-3$ & 0.67 \\
\hline 3201 & $1.19 \mathrm{e}-3$ & 0.58 & $4.50 \mathrm{e}-3$ & 0.57 & $4.76 \mathrm{e}-3$ & 0.58 \\
\hline 6401 & $7.94 \mathrm{e}-4$ & 0.58 & $3.00 \mathrm{e}-3$ & 0.58 & $3.17 \mathrm{e}-3$ & 0.59 \\
\hline 12801 & $4.91 \mathrm{e}-4$ & 0.69 & $1.86 \mathrm{e}-3$ & 0.69 & $1.96 \mathrm{e}-3$ & 0.69 \\
\hline 25601 & $3.17 \mathrm{e}-4$ & 0.63 & $1.20 \mathrm{e}-3$ & 0.63 & $1.26 \mathrm{e}-3$ & 0.64 \\
\hline 51201 & $2.02 \mathrm{e}-4$ & 0.65 & $7.66 \mathrm{e}-4$ & 0.65 & $8.07 \mathrm{e}-4$ & 0.64 \\
\hline 102401 & $1.30 \mathrm{e}-4$ & 0.64 & $4.92 \mathrm{e}-4$ & 0.64 & $5.18 \mathrm{e}-4$ & 0.64 \\
\hline 204801 & $8.29 \mathrm{e}-5$ & 0.65 & $3.14 \mathrm{e}-4$ & 0.65 & $3.30 \mathrm{e}-4$ & 0.65 \\
\hline \hline
\end{tabular}

Table 3: Convergence results for the fully conservative scheme. Shown here are $L_{1}$ errors near the shock for $x \in[-3,3.2]$.

The results for the conservative scheme, shown in table 3 , indicate that for all quantities the convergence near the shock is tending towards $O\left(\Delta x^{2 / 3}\right)$, the expected rate of convergence for a $2^{\text {nd }}$ order high-resolution scheme near contacts [25]. The implication is that the convergence characteristics of the approximations near the contact have influenced the global convergence behavior.

Table 4 shows the $L_{1}$ errors for $x \in[-3.2,-3]$ for the quasi-conservative scheme. Also shown in this table is the increase in total energy over the entire domain. In contrast to the previous results for the conservative scheme, the convergence rate here is tending toward $O(\Delta x)$ for all state variables and the resultant errors are commensurately smaller. That is to say that the quasi-conservative scheme not only appears to provide better qualitative approximations as shown by figures 4 and 5, the measured convergence rate near the shock is more rapid than for the fully conservative scheme. Also notice that the violation of total energy conservation approaches zero at the rate of $O\left(\Delta x^{2 / 3}\right)$ which indicates that the contact dominates the non-conservative process.

Figure 6 shows the density in the computed approximations for all resolutions in tables 3 and 4 in a zoom near the shock. Both the conservative and non-conservative schemes are shown. The quasi-conservative scheme demonstrates more accurate placement of the shock than the fully conservative scheme. For the conservative scheme the number of cells 


\begin{tabular}{||c||c|c||c|c||c|c||c|c||}
\hline \hline$m$ & $e_{\rho}(m)$ & rate & $e_{u}(m)$ & rate & $e_{p}(m)$ & rate & total energy gain & rate \\
\hline \hline 201 & $2.23 \mathrm{e}-3$ & - & $8.45 \mathrm{e}-3$ & - & $8.87 \mathrm{e}-3$ & - & $1.25 \mathrm{e}-1$ & - \\
\hline 401 & $1.77 \mathrm{e}-3$ & 0.33 & $6.73 \mathrm{e}-3$ & 0.33 & $6.96 \mathrm{e}-3$ & 0.35 & $8.05 \mathrm{e}-2$ & 0.63 \\
\hline 801 & $9.73 \mathrm{e}-4$ & 0.86 & $3.70 \mathrm{e}-3$ & 0.86 & $3.84 \mathrm{e}-3$ & 0.86 & $5.15 \mathrm{e}-2$ & 0.64 \\
\hline 1601 & $5.19 \mathrm{e}-4$ & 0.91 & $1.98 \mathrm{e}-3$ & 0.90 & $2.04 \mathrm{e}-3$ & 0.91 & $3.28 \mathrm{e}-2$ & 0.65 \\
\hline 3201 & $2.55 \mathrm{e}-4$ & 1.0 & $9.71 \mathrm{e}-4$ & 1.0 & $1.00 \mathrm{e}-3$ & 1.0 & $2.08 \mathrm{e}-2$ & 0.66 \\
\hline 6401 & $1.24 \mathrm{e}-4$ & 1.0 & $4.72 \mathrm{e}-4$ & 1.0 & $4.89 \mathrm{e}-4$ & 1.0 & $1.32 \mathrm{e}-2$ & 0.66 \\
\hline 12801 & $6.66 \mathrm{e}-5$ & 0.90 & $2.54 \mathrm{e}-4$ & 0.89 & $2.61 \mathrm{e}-4$ & 0.91 & $8.33 \mathrm{e}-3$ & 0.66 \\
\hline 25601 & $3.33 \mathrm{e}-5$ & 1.0 & $1.27 \mathrm{e}-4$ & 1.0 & $1.31 \mathrm{e}-4$ & 0.99 & $5.27 \mathrm{e}-3$ & 0.66 \\
\hline 51201 & $1.65 \mathrm{e}-5$ & 1.0 & $6.32 \mathrm{e}-5$ & 1.0 & $6.50 \mathrm{e}-5$ & 1.0 & $3.32 \mathrm{e}-3$ & 0.67 \\
\hline 102401 & $8.27 \mathrm{e}-6$ & 1.0 & $3.16 \mathrm{e}-5$ & 1.0 & $3.25 \mathrm{e}-5$ & 1.0 & $2.10 \mathrm{e}-3$ & 0.66 \\
\hline 204801 & $4.08 \mathrm{e}-6$ & 1.0 & $1.55 \mathrm{e}-5$ & 1.0 & $1.60 \mathrm{e}-5$ & 1.0 & $1.33 \mathrm{e}-3$ & 0.66 \\
\hline \hline
\end{tabular}

Table 4: Convergence results for the quasi-conservative scheme. Shown here are $L_{1}$ errors near the shock for $x \in[-3,3.2]$. Also shown in this table is the increase in total energy over the entire domain.

by which the numerical shock is misplaced increases as a function of resolution. That is to say that in the limit of vanishing grid spacing, the conservative numerical approximation will misplace the shock by an infinite number of grid cells although the method is convergent in an $L_{1}$ sense. The situation for the non-conservative scheme is much different in that the approximate solutions correctly locate the shock for all resolutions. By this we mean that the analytic shock location lies in the transition region of the approximations for all resolutions. Successive refinement then serves only to sharpen the jump and so the scheme converges as $O(\Delta x)$.

\section{Practical implications}

It is important to appreciate when the issues discussed in section 5 arise in practice and to gain insight as to what types of errors might be expected. To that end we investigate a test problem concerning shock interaction with a two-dimensional cylindrical bubble of higher density material. The single component Euler equations in two dimensions with the JWL equation of state are used with the parameters from table 1 used to define the EOS. The extension of the one-dimensional model to two dimensions is straightforward and can be seen for instance in $[9,16]$. The extension of the numerical methods is also straightforward $[9,10]$.

Figure 7 shows the initial condition we use for this example as a schlieren image and table 5 gives the values for the primitive quantities in the various regions. This initial condition consists of a quiescent state, a shock located at $x=0.05$, and a cylindrical region of gas centered around $(x, y)=(0.5,0)$ with a radius $r=0.25$. The strength of the shock is determined through the pressure rise from $p=1$ in the quiescent gas to $p=1.2$ in the 

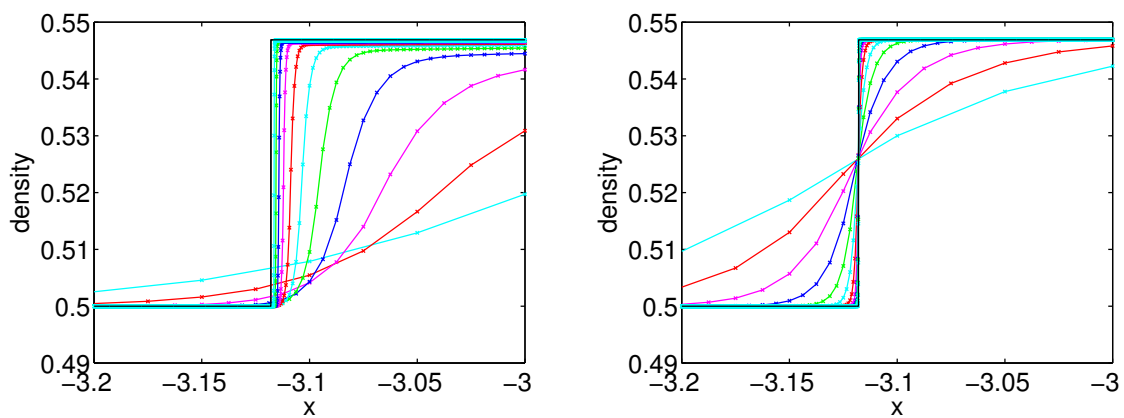

Figure 6: Computed approximations for the density. The black line is the exact solution and the marks the approximations. Numerical results were obtained using the fully conservative scheme (4.2) on the left and the quasi-conservative scheme (4.6) on the right with $m=200,400, \ldots, 204800$.

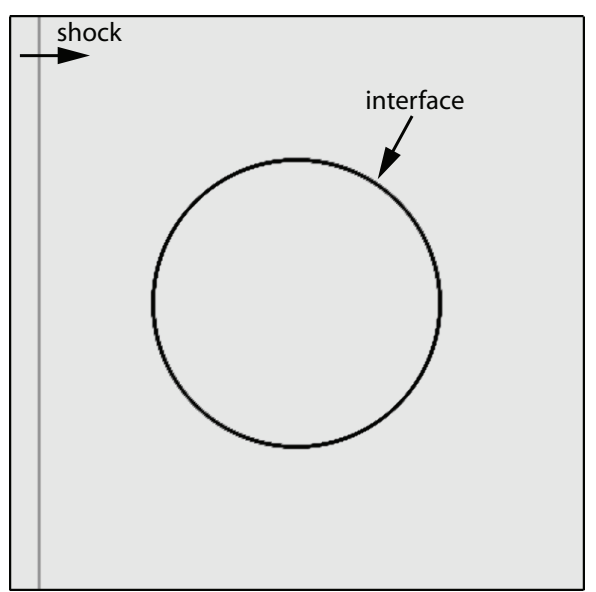

Figure 7: Initial conditions, shown as a numerical schlieren images, for the interaction of a shock with a bubble of higher density material. 


\begin{tabular}{|c||c|c|c|c|}
\hline \hline & $\rho$ & $x$-velocity & $y$-velocity & $p$ \\
\hline \hline post-shock & 0.5505 & 0.1915 & 0 & 1.2 \\
\hline pre-shock & 0.5 & 0 & 0 & 1 \\
\hline bubble & 2 & 0 & 0 & 1 \\
\hline \hline
\end{tabular}

Table 5: Primitive sates for the initial conditions shown in figure 7.

post-shock region and the density and velocity in the post-shock region are determined as in section 3. The computational domain is the unit square $(x, y) \in[0,1] \times[0,1]$ and Neumann boundary conditions are applied everywhere. Adaptive mesh refinement is used as discussed in $[9,19,20]$ and the effective grid resolution at the finest level of refinement is $\Delta x=\Delta y=1 / 400$. The initial condition near the bubble boundary is set by first finding all cells through which the analytic bubble boundary cuts. Each such cell is sub-divided by connecting the two points where the bubble boundary and cell boundary intersect with a straight line and computing the appropriate areas of the resultant regions. The densities from table 5 are then applied over each region and the cell average density computed from this. Finally the pressure and velocity in table 5 are set and a conversion to conserved quantities is performed to complete the initial condition. In this way the initial conditions are set to second order accuracy and each cell along the bubble boundary will have exact cell averaged density based on a many sided polygon.

After some time the shock will interact with the bubble and soon after we view the solution. Figure 8 shows the computed schlieren images and pressure at $t=0.2$ using both the fully conservative and quasi-conservative schemes. The color table for pressure has been chosen to enhance viewing of the higher pressures in the region of shock bubble interaction. As a result the pre- and post-shock regions are clipped out of the color table. The included schlieren images show the broad dynamics and illustrate the basic flow features. As the pressure contours make clear, there are substantial differences in the approximations generated by the two methods. For the fully conservative scheme the pressure jumps through the bubble interface and indeed the location of the interface can be clearly seen as a numerical artifact in the pressure plots. To contrast this, the pressure for the quasi-conservative scheme is smooth through the interface and as a result the overall solution character matches much more closely with expected results based on simulations with ideal gases $[5,9]$.

To more clearly see the behavior of the simulation results, figure 9 shows the pressure and $x$-velocity along the horizontal line through the bubble center. From this figure it is clear that the quasi-conservative scheme maintains a smooth pressure profile through the material interface, but that the fully-conservative scheme contains a small oscillation. All evidence indicates that this oscillation has similar origins to the oscillations found in section 5 and we therefore conclude that they represents unphysical behavior.

Figure 10 shows the numerical schlieren and pressure contour for the two simulations 

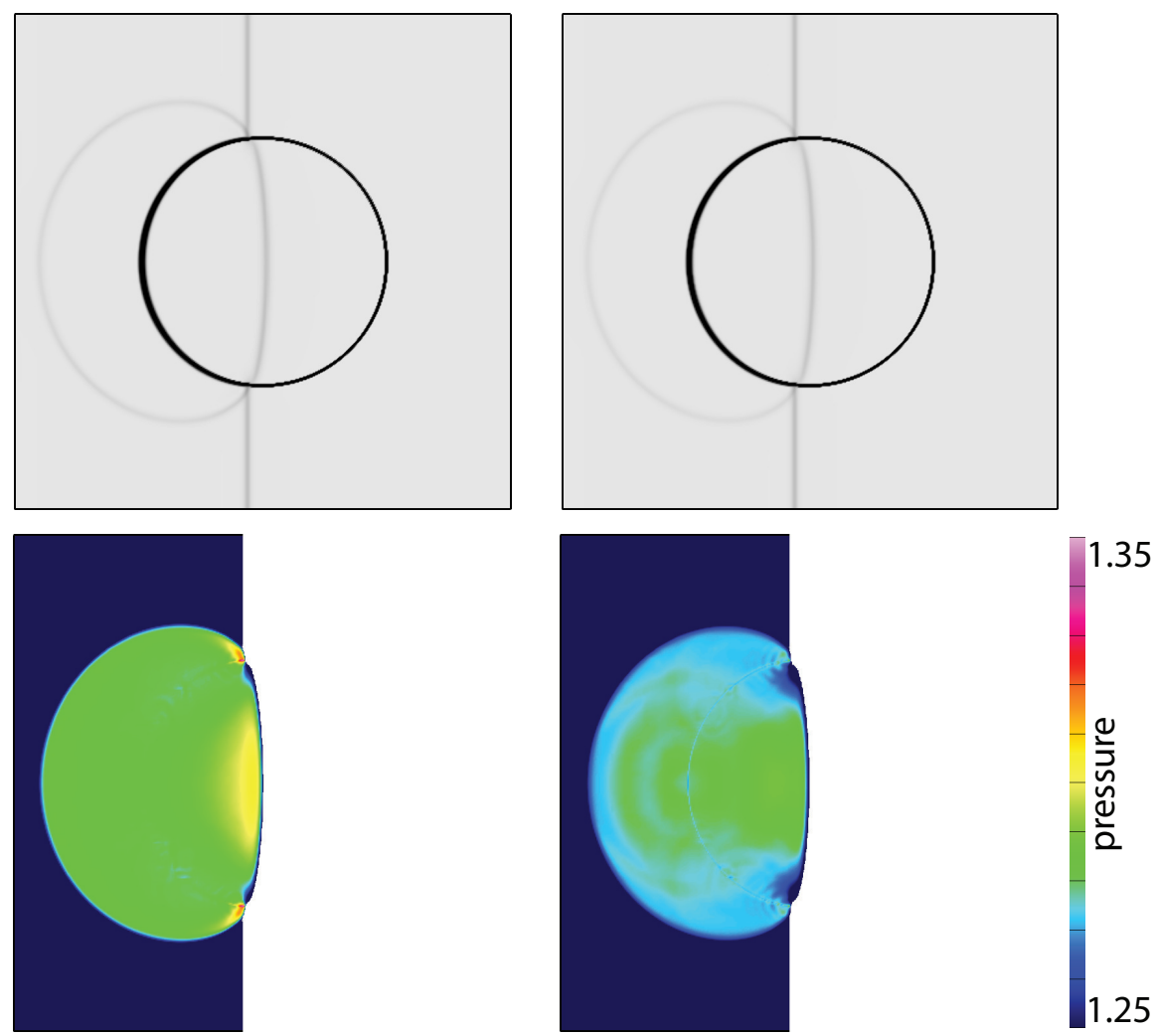

Figure 8: Simulation results for the shock-bubble interaction problem at $t=0.2$. The upper images show a numerical schlieren and the lower images the pressure. The results for the quasi-conservative scheme are on the left and the fully conservative scheme on the right.
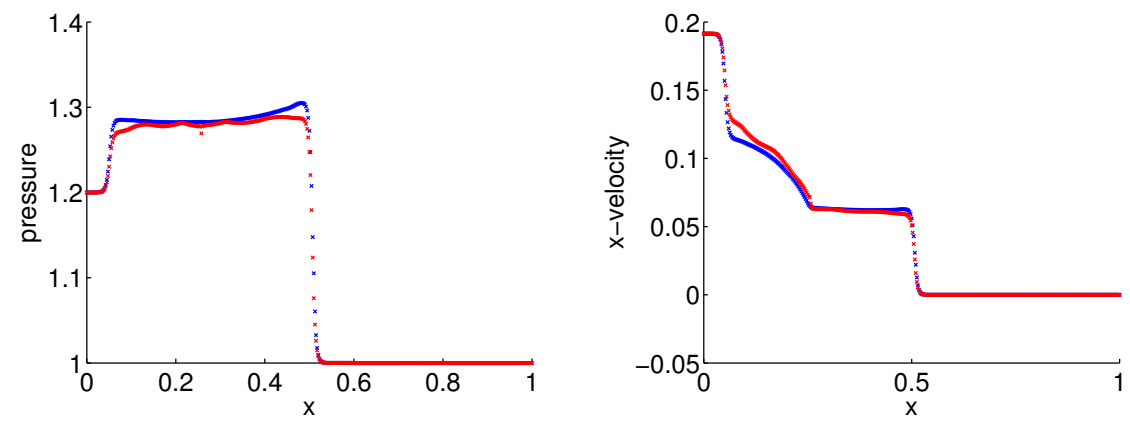

Figure 9: Pressure and $x$-velocity along the horizontal line through the bubble center at $t=0.2$. The red marks indicate the fully conservative results and the blue marks the quasi-conservative ones. 

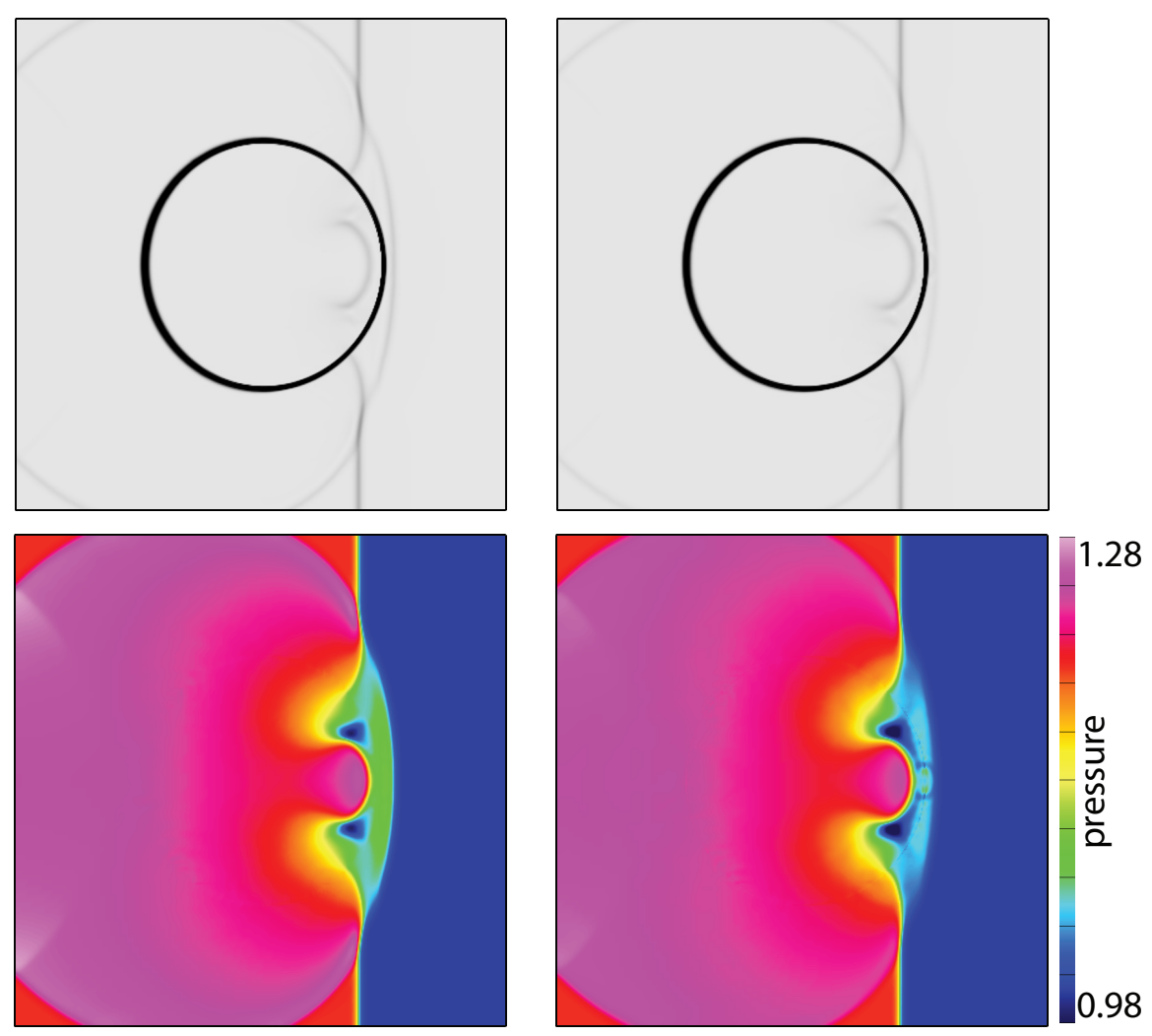

Figure 10: Simulation results for the shock-bubble interaction problem at $t=0.31$. The upper images show a numerical schlieren and the lower images the pressure. The results for the quasi-conservative scheme are on the left and the fully conservative scheme on the right.

at the slightly later time $t=0.31$. Here the shock has nearly completed its transit of the bubble and many complex interactions of the reflected shocks, transmitted shocks, and the bubble interface have take place. The first item to notice is again how the pressure plot for the fully conservative scheme shows the bubble boundary where the pressure oscillates as the interface diffuses. This is particularly true near the far right boundary of the bubble showing in light blue to green in the pressure contours. As before this is interpreted as unphysical behavior. Also of concern is that the interaction of the waves with the boundary has resulted in different qualitative solutions. For instance the approximation resulting from the quasi-conservative scheme has the transmitted shock emerging from the bubble boundary and strengthening the diffracted shock. This shows as a light green in the color contour plot pressure. To contrast this, the same shock structure is significantly weaker for the fully-conservative case. To bring this point home, figure 11 shows the pressure and $x$-velocity along the horizontal line through the bubble center at $t=0.31$. Here again notice that the quasi-conservative scheme not only lacks unphysical wiggles through the bubble interface, but also that the shock structures are stronger for these results. It is 

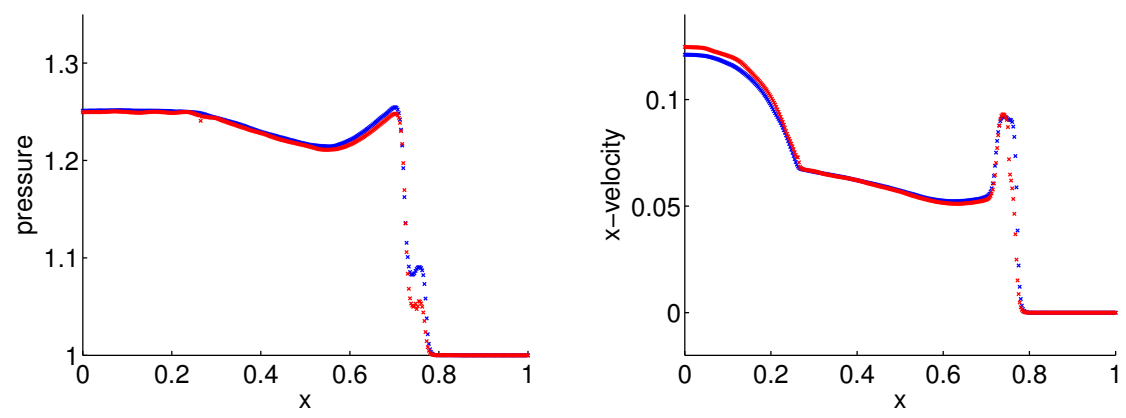

Figure 11: Pressure and $x$-velocity along the horizontal line through the bubble center at $t=0.31$. The red marks indicate the fully conservative results and the blue marks the quasi-conservative ones.

difficult to definitively declare that one result is "correct", but all evidence indicates that the results from the fully conservative scheme should not be trusted.

\section{Conclusions}

In this paper we have presented a single fluid-model with non-ideal equations of state and shown that numerical approximation by a standard fully conservative computational method may produce poor results. Inclusion of a non-conservative source term was motivated by a contact only problem. A new switching mechanism was also devised and shown to be necessary in order to ensure convergence to a conservative weak solution. A simple Riemann problem then demonstrated that the resulting quasi-conservative scheme produces results which do not contain the unphysical numerical artifacts seen in the fully conservative counterpart. Furthermore it was shown that the order of convergence near the shock was higher for the quasi-conservative scheme than for the fully conservative scheme. This result stands somewhat in contrast to conventional wisdom which says that conservative schemes should perform better in terms of shock location than non-conservative schemes. To further address the concerns over conservation in the quasi-conservative scheme, it was shown that the violation of energy conservation approaches zero as the mesh is refined and a conservative result is obtained in the limit.

In the end, the primary concern should be that a given simulation converge rapidly to the physically relevant solution. In some sense, conservation is nothing more than another governing equation and there is no particular reason why it should be satisfied exactly for all resolutions. More effective is to satisfy the whole set of equations, including conservation, to some order and rely on convergence in the limit of small mesh spacing. The high-resolution methods investigated in this paper have asymptotically identical truncation error and so in that sense they are equivalent. However, it is clear that some methods converge more rapidly to the relevant solution than do other methods and it should be rapid convergence as well as physical relevance that is most important. With this in 
mind, a more practical realization of the potential problems was investigated. Here the interaction of a shock with a 2-D cylindrical bubble of dense gas was investigated. The fully conservative method shows results which have obvious problems in that the pressure oscillates through the bubble interface while the quasi-conservative results are in accord with expectations. Based on the information at hand we are drawn to the conclusion that the approximations from the quasi-conservative method are the ones to be believed. It should also be stated that although the variation between the results from the different solution methods is relatively small, the addition of further physical processes can amplify any differences which do exist. Chemical mechanism, for example, are often quite stiff as well as highly non-linear and any differences in simulation results from the flow solvers can result in substantial solution differences in this regime.

\section{Acknowledgements}

This study has been supported by Lawrence Livermore National Laboratory under the auspices of the U.S. Department of Energy through contract number DE-AC52-07NA27344.

\section{References}

[1] P. Lax, B. Wendroff, Systems of conservation laws, Commun. Pur. Appl. Math. 13 (1960) 217-237.

[2] T. Y. Hou, P. G. Le Floch, Why nonconservative schemes converge to wrong solutions: Error analysis, Math. Comp. 62 (206) (1994) 497-530.

[3] J. Glimm, Solutions in the large for nonlinear hyperbolic systems of equations, Commun. Pur. Appl. Math. 18 (1965) 697-715.

[4] S. Karni, Multicomponent flow calculations by a consistent primitive algorithm, J. Comput. Phys. 112 (1994) 31-43.

[5] J. J. Quirk, S. Karni, On the dynamics of a shock-bubble interaction, J. Fluid Mech. 318 (1996) 129-163.

[6] R. Abgrall, How to prevent pressure oscillations in mulicomponent flow calculations: A quasi conservative approach, J. Comput. Phys. 125 (1996) 150-160.

[7] R. Saurel, R. Abgrall, A simple method for compressible multifluid flows, SIAM J. Sci. Comput. 21 (3) (1999) 1115-1145.

[8] R. Abgrall, S. Karni, Computations of compressible multifluids, J. Comput. Phys. 169 (2001) 594-623.

[9] J. W. Banks, D. W. Schwendeman, A. K. Kapila, W. D. Henshaw, A high-resolution Godunov method for compressible multi-material flow on overlapping grids, J. Comput. Phys. 223 (2007) 262-297.

[10] J. W. Banks, W. D. Henshaw, D. W. Schwendeman, A. K. Kapila, A study of detonation propagation and diffraction with compliant confinement, Combust. Theory and Modelling 12 (4) (2008) 769-808.

[11] R. Saurel, E. Franquet, E. Daniel, O. L. Metayer, A relaxation-projection method for compressible flows. Part I: The numerical equation of state for the Euler equations, J. Comput. Phys. 223 (2007) 822-845.

[12] B. M. Dobratz, Properties of chemical explosives and explosive simulants, Tech. Rep. LLNL UCRL-51319, Lawrence Livermore Laboratory National Laboratory (July 1974). 
[13] R. Menikoff, B. J. Plohr, The Riemann problem for fluid flow or real materials, Reviews of Modern Physics 61 (1) (1989) 75-130.

[14] M. Larini, R. Saurel, J. C. Loraud, An exact Riemann solver for detonation products, Shock Waves 2 (1992) 225-236.

[15] R. Saurel, M. Larini, J. C. Loraud, Exact and approximate Riemann solvers for real gases, J. Comput. Phys. 112 (1994) 126-137.

[16] G. B. Whitham, Linear and Nonlinear Waves, Wiley-Interscience, New York, 1974.

[17] U. M. Ascher, L. R. Petzold, Computer Methods for Ordinary Differential Equations and Differential-Algebraic Equations, SIAM, Philadelphia, 1998.

[18] A. K. Kapila, D. W. Schwendeman, J. B. Bdzil, W. D. Henshaw, A study of detonation diffraction in the Ignition-and-Growth model, Combust. Theory and Modelling 11 (5) (2007) 781-822.

[19] W. D. Henshaw, D. W. Schwendeman, An adaptive numerical scheme for high-speed reactive flow on overlapping grids, J. Comput. Phys. 191 (2) (2003) 420-447.

[20] W. D. Henshaw, D. W. Schwendeman, Moving overlapping grids with adaptive mesh refinement for high-speed flow, J. Comput. Phys. 216 (2) (2006) 744-779.

[21] S. K. Godunov, Finite difference method for numerical computation of discontinuous solutions of the equations of fluid dynamics, Mat. Sb. 47 (1959) 271-306.

[22] B. van Leer, Towards the ultimate conservative difference scheme, V. A second-order sequel to Godunov's method, J. Comput. Phys. 32 (1979) 101-136.

[23] E. F. Toro, Riemann Solvers and Numerical Methods for Fluid Dynamics, Springer, Berlin, 1999.

[24] P. Glaister, An approximate linearised riemann solver for euler equations for real gases, J. Comput. Phys. 74 (1988) 382-408.

[25] J. W. Banks, T. Aslam, W. J. Rider, On sub-linear convergence for linearly degenerate waves in capturing schemes, J. Comput. Phys. 227 (14) (2008) 6985-7002. 